\title{
3D-Biaural Localization of A Stationary Random Acoustical Source in Near-Field by Using Amplitude Attenuation of Wave Propagation and An Additional Rotation of Detectors
}

\author{
Kimio SASAKI* and Kohji UCHINO** \\ * Institute of Engineering Mechanics, University ** Technical Operations' Center, Nippon TV \\ of Tsukuba, Ten-Ohdai 1-1-1, Tsukuba-shi, Network Co., 2 Banchyo 14, Chiyoda-ku, \\ Ibaraki 305, Japan \\ Tokyo 102-40, Japan
}

\begin{abstract}
With the aims of exploring humankind's biaural localization ability as well as developing refined devices for environmental recognition in robotics, a practical method for 3D-biaural localization of a stationary random acoustical source in near-field is proposed by newly introducing a rotation of detectors, in addition to the previously proposed method using amplitude attenuation as well as the conventional relative time delay between detected signals. The principle of the proposed $3 D$-localization and theoretical evaluation of the position estimate deriverd from auto- and cross-spectral analyses of detected signals are presented, together with the results of numerical analysis, illustrating the effectiveness as well as fundamental characteristics of this method under practical circumstances.
\end{abstract}

\section{INTRODUCTION}

3D-biaural localization of a random acoustical source in near-field is an important task for exploring humankind's biaural localization ability as well as for developing refined devices for environmental recognition in robotics.

In the previous paper [1], we proposed a practical method of simultaneous estimation of the range and bearing of a stationary planer random acoustical source in near-field, by newly detecting information about amplitude attenuation of wave propagation and combining it with the conventional relative time delay between detected signals, which is the only available information in most conventional schemes for far-field sources [2], [3]. As made clear, however, all the that can be estimated in any biaural localization schemes are equivalently reduced to the two distances between detectors and the source. As the result, the full position estimation is possible in the plane, but not in the 3D-space.

In this paper, we propose a practical method for 3D-biaural localization of the source, which can estimate the position in the 3D-space, by additionally introducing a rotation of detectors to the previously proposed method, in order to simulate neck mevement of a human [4].

In the followings, are presented a geometry and mathematical model of the problem, the principle of the proposed simultaneous estimation of the range and bearings, statistical properties of the estimates, expected source distribution area in 3D-space and cross-sectional biaural localizability in 2D-space and the results of numerical analysis under assumed practical conditions, illustrating the effectiveness of the proposed method as well as its fundamental characteristics.

\section{GEOMETRY AND MATHEMATICAL MODEL OF THE BIAURAL LOCALIZATION}

Consider a geometry of biaural detection in Fig. 1. With aid of detector rotation around the $z$-axis by an angle $\alpha$, a stationary Gaussian random signal $s(t)$ with zero-mean and power spectrum $\Phi s s(f)$ from a stationary point source $P$ is received twice at two microphones $M i$ s and $M i$ 's in the $(x, y)$ plane before and after the rotation, respectively, with the spacing between the microphones being kept as $2 d$. The range $r o$ and bearings $\phi$ and $\theta$ of the source position to be estimated are defined as the polar co-ordinates of the source $P$. According to the previous paper [1], let us postulate the following assumptions for simplification of analysis.

Assumptions;

(1) Microphones' separation $2 d$ : small and almost the same as the spacing between a human's ears.

(2) Wave propagation: spherical

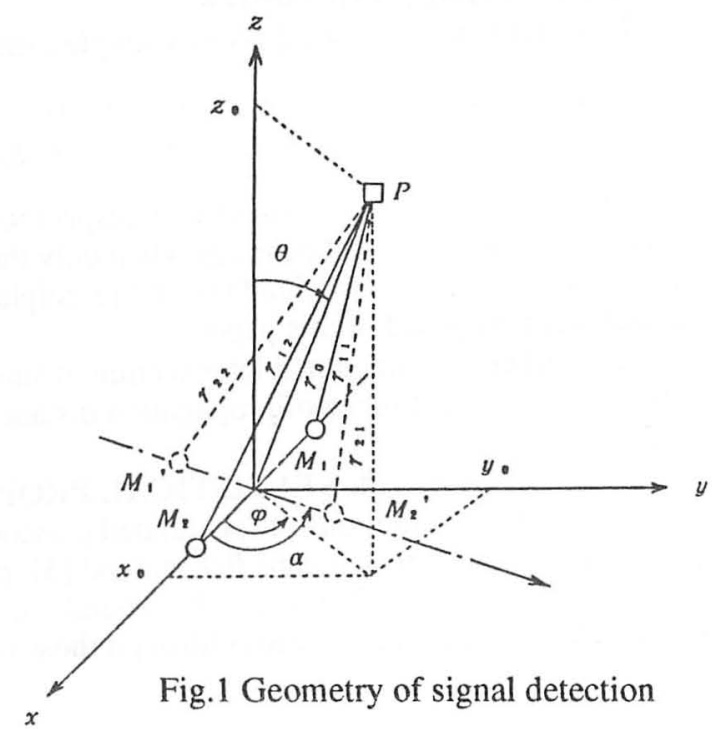

Fig.1 Geometry of signal detection 
(3) Additive noises at two microphones: Gaussian, independent each other as well as to s(t), and have the same power spectrum $\Phi_{n n}(f)$.

(4) Observation time $T$ : long enough for precise power spectral estimation by standard means such as $B$ - $T$ method.

\section{PRINCIPLE OF SIMULTANEOUS ESTIMATION OF THE RANGE AND BEARINGS}

Under the above assumptions, detected signals at two microphones can be directly expressed by Eq. (1), where suffix $i=1$ and 2 represent the states before and after the detector rotation, respectively, and $r_{i j}, D_{i j}$ and $n i j(t)$ are the distance from $P$ to $M j$, the corresponding time delay and additive noise at $M j$, respectively. Hence the associated autoand cross-power spectra of /between detected signals can be written by Eqs. ( $2 \mathrm{a})$ and ( $2 \mathrm{~b})$ as follows:

$$
\begin{array}{rrr}
x_{i j}(t)=r_{i j}{ }^{-1} \cdot s\left(t-D_{i j}\right)+n_{i j}(t), & i, j=1,2(1) & \Phi_{x i 1 \times i 2}(f)=\left(r_{i 1} r_{i 2}\right)^{-1} \cdot \Phi_{s s}(f) \\
\Phi_{x i j \times i j}(f)=r_{i j}{ }^{-2} \cdot \Phi_{s s}(f)+\Phi_{n n}(f), & i, j=1,2(2 \mathrm{a}) & \operatorname{xexp}\left[-j 2 \pi f\left(D_{i 2}-D_{i 1}\right)\right], \quad i=1,2
\end{array}
$$

Therefore, as discussed in the previous paper [1], two sets of information about the difference of propagation distances and amplitude attenuation of propagated waves are obtained from these spectra. Since each of the information pairs are functions of propagation distances, these spectral relations provide two sets of propagation distances. That is, for each $i$ corresponding to one of the states before and after the detector rotation, the phase of cross-power spectral estimate $\Phi x i 1 x i 2(f)$ directly gives the information about the difference of propagation distances $r d i=r i 1-r i 2$,

$$
\hat{r}_{d i}(f)=c_{0} /(2 \pi f) \cdot \tan ^{-1}\left\{\operatorname{Im}\left[\hat{\Phi}_{x i 1 x i 2}(f)\right] / \operatorname{Re}\left[\hat{\Phi}_{x i 1 x i 2}(f)\right]\right\}=\hat{r}_{i 1}-\hat{r}_{i 2},
$$

while the difference of the auto-power spectral estimates $\Phi$ xijxij $(f)$ s divided by the modulus of the cross-power spectral one provides the information about amplitude attenuation of wave propagation without any effects of the power spectra due to the source signal as well as additive noises, since they are expected to be cancelled out completely as follows:

$$
\hat{B}_{i}(f)=\left[\widehat{\Phi}_{x i 2 \times i 2}(f)-\widehat{\Phi}_{x i 1 x i 1}(f)\right] /\left|\hat{\Phi}_{x i 1 \times i 2}(f)\right|=\hat{r}_{i 1} / \hat{r}_{i 2}-\hat{r}_{i 2} / \hat{r}_{i 1} \text {, }
$$

In the above, $A$ denotes an estimate of a quantity $A$ and $c o$ is the wave velocity.

By combining Eqs. ( $3 a)$ and ( $3 b$ ) with the next geometric relations

$$
\begin{array}{ll}
r_{i 1}{ }^{2}+r_{i 2}{ }^{2}=2\left(r_{0}{ }^{2}+d^{2}\right), \quad i=1,2 & \text { (4a) } \\
r_{11}{ }^{2}-r_{12}{ }^{2}=4 r_{0} d \sin \theta \cos \varphi, & \text { (4b) }
\end{array} \quad r_{21}{ }^{2}-r_{22}{ }^{2}=4 r_{0} d \sin \theta \cos (\varphi-\alpha),
$$

two sets of propagation distances $r_{i j}(i, j=1,2)$ can be estimated as mentioned previously, resulting in acquisition of four parameters about the source position, which is uniquely determined only by the three in the 3D-space. From the general viewpoint, a plausible candidate for the position estimate will be obtained by some means such as $L M S$ method, but we made clear, in the previous paper, the necessity to estimate the range more precisely than the bearing since the range estimate is more sensitive to estimation errors of the spectra and/or additive noises than the bearings. So, the more precise position estimation can be expected if we first estimate the range by an arithmetic mean of the raw estimates derived from Eq. (4a) for $i=1$ and 2 ,

$$
\hat{r}_{0}(f)=\frac{1}{2} \cdot \sum_{i=1}^{2}\left\{\hat{r}_{d i}{ }^{2}(f)\left\{\hat{B}_{i}^{2}(f)+4+2\left[\hat{B}_{i}^{2}(f)+4\right]^{1 / 2}\right\}\left[2 \hat{B}_{i}^{2}(f)\right]^{-1}-d^{2}\right\}^{1 / 2},
$$

and then, by use of it, the bearing estimate $\phi(f)$ is obtained by solving $\cos (\hat{\varphi}-\alpha) \hat{r}_{d_{1}}{ }^{2}(f) \hat{K}_{1}(f)=\cos \hat{\varphi} \hat{r}_{d 2}{ }^{2}(f) \hat{K}_{2}(f)$, where $K i(f)=2+\left[B i(f)^{2}+4\right]^{1 / 2}(i=1,2)$.

Especially, taking $\alpha=\pi / 2$ gives a simple estimate $\phi(f)$, together with another bearing estimate $\theta(f)$ as follows:

$$
\begin{aligned}
& \hat{\varphi}(f)=\tan ^{-1}\left\{\hat{r}_{d 2}{ }^{2}(f) \hat{B}_{1}(f) \hat{K}_{2}(f) /\left[\hat{r}_{d 1}{ }^{2}(f) \hat{B}_{2}(f) \hat{K}_{1}(f)\right]\right\}, \\
& \hat{\theta}(f)=\sin ^{-1}\left\{\left[\hat{r}_{d 1}{ }^{4}(f) \hat{B}_{2}{ }^{2}(f) \hat{K}_{1}{ }^{2}(f)+\hat{r}_{d 2}{ }^{4}(f) \hat{B}_{1}{ }^{2}(f) \hat{K}_{2}{ }^{2}(f)\right]^{1 / 2} /\left[4 \hat{r}_{0}(f) d \hat{B}_{1}(f) \hat{B}_{2}(f)\right]\right\},(5 \mathrm{c})
\end{aligned}
$$

These relations are pointwise with respect to frequency $f$, as is explicitly indicated above, and lead to the effective estimates of the range and bearings when only the frequency content of high SNR (signal-to-noise ratio) in the detected signals is used. This is the fundamental principle of the 3D-biaural localization of a stationary random acoustical source in near-field proposed in this paper.

As will be examined in a later section, it should be noted that the final estimates are highly nonlinear with respect to estimates of the difference of propagation distances and amplitude attenuation of wave propagation in the near-field.

\section{STATISTICAL PROPERTIES OF THE PROPOSED ESTIMATES}

Under the assumptions (4) postulated previously, all the auto- and cross-power spectra of the detected signals can be precisely estimated by standard $B-T$ method [5], resulting in the good range and bearing estimates. Thus, by linearizing $r d i(f)$ and $B i(f)$ in Eqs.(3a) and (3b) around their true values with respect to every power spectral estimate, we can evaluate their sampling variations through those of power spectral estimates as follows: 


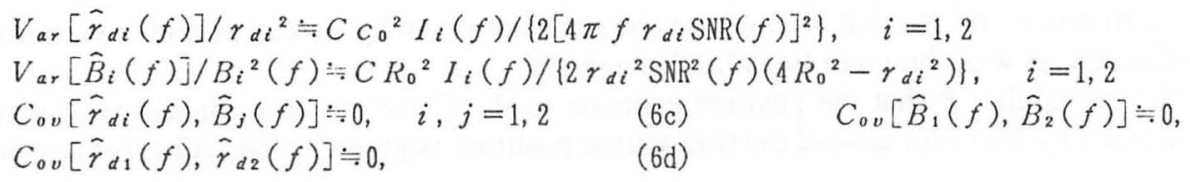

$(6 a)$

(6e)

where $R o^{2}=r o^{2}+d^{2}, S N R(f)=\Phi s s(f) / \Phi n n(f), I i(f)=8 R o^{2} S N R(f)+\left(2 R o^{2}-r d i^{2}\right)(i=1,2)$ and $C=E w / T$, i.e., ratio of used window energy to data length. Eqs.(6a) - (6c) are the same as Eqs.(12a) - (12c) in Ref.[1], and Eqs.(6d) and (6e) are derived on the basis of independent observations before and after the detector rotation.

Therefore, by inserting these results into the similarly linearized version of range and bearing estimates in Eqs.(5a) through $(5 \mathrm{c})$, we obtain the following sampling characteristics of the proposed estimates:

$$
\begin{aligned}
& V_{a r}\left[\hat{r}_{0}(f)\right] \fallingdotseq C R_{0}{ }^{4} /\left[32 r_{0}{ }^{2} \operatorname{SNR}^{2}(f)\right] \cdot \sum^{2} I_{i}(f) J_{i}(f) / r_{d i}{ }^{2}, \\
& V_{a r}[\hat{\varphi}(f)] \fallingdotseq C \cos ^{4} \varphi \cot ^{2} \varphi\left(r_{d 2} / r_{d 1}\right)^{i}\left(R_{d 2}{ }^{2}+R_{y d^{2}}\right)^{2} /\left[2 \operatorname{SNR}^{2}(f)\left(R_{d 1}{ }^{2}+R_{x d^{2}}{ }^{2}\right)\right] \\
& X\left\{I_{1}(f) / r_{d 1}{ }^{2} \cdot\left[\left(c_{0} / 2 \pi f\right)^{2}+R_{d 1}{ }^{4} /\left(R_{10}{ }^{2} R_{x d^{4}}\right)\right]\right. \\
& \left.+I_{2}(f) / r_{d 2}^{2} \cdot\left[\left(c_{0} / 2 \pi f\right)^{2}+R_{d 2}{ }^{4} /\left(R_{20}{ }^{2} R_{y d^{4}}\right)\right]\right\}, \\
& V_{a r}[\hat{\theta}(f)] \fallingdotseq C I_{1}(f) \tan ^{2} \theta /\left[2 r_{0}{ }^{2} r_{d 1} \operatorname{SNR}^{2}(f)\right] \cdot\left\{\left(\left[\left(3 r_{0}{ }^{2}-d^{2}\right) c_{0} / 2 \pi f\right]^{2}+R_{0}{ }^{4} R_{d 1}{ }^{4} /\left(R_{10}{ }^{2} R_{x d^{4}}\right)\right.\right. \\
& \left.\mathrm{x}\left[\left(R_{0} R_{d_{1}} / R_{x d}\right)^{2}-\left(3 \gamma_{0}^{2}-d^{2}\right)\right]^{2}\right) / 8-2\left(r_{0} \gamma_{d 2} \cos \varphi / r_{d 1}\right)^{4} \cdot\left(R_{d 2}^{2}+R_{y d^{2}}\right)^{2}\left[\left(c_{0} / 2 \pi f\right)^{2}+\right. \\
& \left.+R_{d 1}{ }^{4} /\left(R_{10}{ }^{2} R_{x d^{4}}\right)\right] /\left(R_{d 1}{ }^{2}+R_{x d^{2}}\right)^{2}-\left(r_{0} \sin \varphi\right)^{2}\left(\left[\left(3 r_{0}{ }^{2}-d^{2}\right) c_{0} / 2 \pi f\right]^{2}+\right. \\
& \left.\left.+R_{0}{ }^{4} R_{d 1}{ }^{4}\left[3 r_{0}^{2}-d^{2}-\left(R_{0} R_{d 1} / R_{x d}\right)^{2}\right] /\left(R_{10}{ }^{2} R_{x d^{4}}\right)\right)\right\} \\
& +C I_{2}(f) \tan ^{2} \theta /\left[2 r_{0}{ }^{2} \gamma_{d 2} \operatorname{SNR}^{2}(f)\right] \cdot\left\{R_{0}{ }^{4} / 8 \cdot\left[\left(C_{0} / 2 \pi f\right)^{2}+R_{0}{ }^{4} R_{d 2}{ }^{4}\left(R_{d 2}{ }^{2}+R_{y d^{2}}\right)^{2} /\left(R_{10}{ }^{2} R_{y d^{4}}\right)\right]\right. \\
& +2 r_{0}{ }^{4} \cos ^{4} \varphi / \gamma_{d 1}{ }^{2} \cdot\left(R_{d 2}{ }^{2}+R_{y d^{2}}{ }^{2}\right)^{2} \times\left[\left(c_{0} / 2 \pi f\right)^{2}+R_{d 2}{ }^{4} /\left(R_{20}{ }^{2} R_{y d}{ }^{4}\right)\right] /\left(R_{d 1}{ }^{2}+R_{x d^{d}}\right)^{2} \\
& \left.-\left(r_{0} R_{0} \sin \varphi\right)^{2}\left[\left(c_{0} / 2 \pi f\right)^{2}+R_{0}{ }^{4} R_{d 2}{ }^{4}\left(R_{d 2}{ }^{2}+R_{y d^{2}}\right) /\left(R_{20}{ }^{2} R_{y d^{2}}{ }^{2}\right)\right]\right\} \text {, } \\
& C_{0 v}\left[\hat{r}_{0}(f), \hat{\varphi}(f)\right] \fallingdotseq C R_{0}{ }^{2} \sin \varphi \cos \varphi /\left[8 r_{0} \operatorname{SNR}^{2}(f)\right] \\
& \times\left\{I_{2}(f) / r_{d 2}{ }^{2} \cdot\left[\left(c_{0} / 2 \pi f\right)^{2}-r_{d 2}{ }^{2} R_{0}{ }^{4} R_{d 2}{ }^{4}\left(R_{d 2}{ }^{2}+R_{y d}{ }^{2}\right) /\left(R_{20}{ }^{2} R_{y d}{ }^{6}\right)\right]\right\} \\
& \left.-I_{1}(f) / \gamma_{d 1}{ }^{2} \cdot\left[\left(c_{0} / 2 \pi f\right)^{2}+\gamma_{d 1}{ }^{2} R_{0}{ }^{4} R_{d 1}{ }^{4}\left(R_{d 1}{ }^{2}+R_{x d^{2}}{ }^{2}\right) /\left(R_{10}{ }^{2} R_{x d^{6}}\right)\right]\right\} \text {, } \\
& C_{0 v}\left[\hat{\gamma}_{0}(f), \hat{\theta}(f)\right] \doteqdot C R_{0}{ }^{2} \tan \theta /\left[32 r_{0}{ }^{3} \operatorname{SNR}^{2}(f)\right] \cdot\left\{I_{1}(f) / r_{d 1}{ }^{2} \cdot\left(\left(3 r_{0}{ }^{2}-d^{2}\right)\left(c_{0} / 2 \pi f\right)^{2}-\right.\right. \\
& \left.-R_{0}{ }^{4} R_{d 1}{ }^{4}\left(R_{d 1}{ }^{2}+R_{x d^{2}}{ }^{2}\right)\left[R_{0}{ }^{2} R_{d 1}{ }^{2}-\left(3 \gamma_{0}{ }^{2}-d^{2}\right) R_{x d^{2}}{ }^{2}\right] /\left(R_{10}{ }^{2} R_{x d^{8}}{ }^{8}\right)\right)-I_{2}(f) / r_{d 2}{ }^{2} \text {. } \\
& \left.\mathrm{x}\left[\left(c_{0} / 2 \pi f\right)^{2}-R_{0}{ }^{4} R_{d 2}{ }^{4}\left(R_{d 2}{ }^{2}+R_{y d^{2}}\right)^{2} /\left(R_{20}{ }^{2} R_{y d}{ }^{8}\right)\right]\right\}-C R_{0}{ }^{2} \tan ^{2} \theta \sin ^{2} \varphi /\left[8 r_{0} \operatorname{SNR}^{2}(f)\right] \cdot \\
& \times\left\{I_{1}(f) / r_{d 1}{ }^{2} \cdot\left[\left(c_{0} / 2 \pi f\right)^{2}+R_{0}{ }^{4} R_{d 1}{ }^{4}\left(R_{d 1}{ }^{2}+R_{x d^{2}}{ }^{2}\right) /\left(R_{10}{ }^{2} R_{x d^{6}}{ }^{6}\right)\right]\right. \\
& \left.-I_{2}(f) / \gamma_{d 2}{ }^{2} \cdot\left[\left(c_{0} / 2 \pi f\right)^{2}+R_{0}{ }^{4} R_{d 2}{ }^{4}\left(R_{d 2}{ }^{2}+R_{y d}{ }^{2}\right) /\left(R_{20}{ }^{2} R_{y d}{ }^{6}\right)\right]\right\} \text {, } \\
& C_{o v}[\hat{\varphi}(f), \hat{\theta}(f)] \fallingdotseq C \tan \theta \sin \varphi \cos \varphi I_{1}(f) /\left[8 r_{0}{ }^{2} r_{d 1}{ }^{2} \operatorname{SNR}^{2}(f)\right] \cdot\left(\left[3 r_{0}{ }^{2}-d^{2}-\left(2 r_{0} \sin \varphi\right)^{2}\right] \cdot\right. \\
& \left.\mathrm{x}\left(c_{0} / 2 \pi f\right)^{2}+R_{0}{ }^{2} R_{d 1}{ }^{4}\left[1-\left(2 r_{0} \sin \varphi\right)^{2} R_{x d^{2}}{ }^{2}\right] /\left(R_{10}{ }^{2} R_{x d^{6}}\right)\right)-C \tan \theta \sin \varphi \cos \varphi / \\
& /\left[8 r_{0}{ }^{2} r_{d 2}{ }^{2} \operatorname{SNR}^{2}(f)\right] \cdot\left(\left[R_{0}{ }^{2}-\left(2 r_{0} \sin \varphi\right)^{2}\right]\left(c_{0} / 2 \pi f\right)^{2}+R_{0}{ }^{4} R_{d 2}{ }^{4}\right. \text {. } \\
& \left.\times\left\{R_{0}{ }^{2} R_{d 2}{ }^{2}+\left[R_{0}^{2}-\left(2 r_{0} \sin \varphi\right)^{2}\right] R_{y d^{2}}{ }^{2}\right\} /\left(R_{20}{ }^{2} R_{y d^{6}}\right)\right),
\end{aligned}
$$

In the above, the next abbreviations are used for simplicity and given space.

$$
\begin{aligned}
J_{i}(f) & \equiv\left(c_{0} / 2 \pi f\right)^{2}+4 R_{0}{ }^{2}-\gamma_{d i}{ }^{2}, \quad i=1,2 \\
R_{d i}{ }^{2} & \equiv 2 R_{0}{ }^{2}-r_{d i}{ }^{2}, \quad i=1,2 \\
R_{i 0}{ }^{2} & \equiv 2 R_{0}{ }^{2}+R_{d i}{ }^{2}, \quad i=1,2
\end{aligned}
$$

$R_{x d^{4}} \equiv\left(4 r_{0} d \sin \theta \cos \varphi\right)^{2}+R_{d 1}{ }^{2}$

$R_{y d^{4}} \equiv\left(4 r_{0} d \sin \theta \sin \varphi\right)^{2}+R_{d 2}^{2}$.

Relations Eqs.(7a) through (7f) are the fundamental sampling characteristics of the proposed estimates and serve as the basis for evaluating the expected 3D-distribution area of the random acoustical source and for deriving crosssectional biaural localizability in two dimensions by the proposed method, as will be examined in the following sections.

\section{3D-EXPECTED SOURCE DISTRIBUTION AREA AND CROSS-SECTIONAL BIAURAL \\ LOCALIZABILITY}

Under the assumptions, the proposed range and bearing estimates are expected to be unbiased and Gaussian, as long as the applied linearization remains valid. Thus, transforming the estimation error vector $\boldsymbol{e}$ from polar co-ordinates $(r, \phi, \theta)$ to $\varepsilon$ in the Cartesian frame $(x, y, z)$ with aid of a matrix $\boldsymbol{H}$ defined by Eq.(9a), provides a 3D-Gaussian PDF (probability density function) $p(\varepsilon)$ as follows:

$$
H=\left(\begin{array}{ccc}
\sin \theta \cos \varphi & r_{0} \cos \theta \cos \varphi & -r_{0} \sin \theta \sin \varphi \\
\sin \theta \sin \varphi & r_{0} \cos \theta \sin \varphi & r_{0} \sin \theta \cos \varphi \\
\cos \theta & -r_{0} \sin \theta & 0
\end{array}\right)
$$

$$
\begin{aligned}
p(\varepsilon) & =\left[(2 \pi)^{3} \cdot \operatorname{det}\left(R_{\varepsilon}\right)\right]^{-1 / 2} \exp \left(-\varepsilon^{\tau} R_{\varepsilon}^{-1} \varepsilon / 2\right) \\
R_{\varepsilon} & =H R_{e} H^{\tau}
\end{aligned}
$$


where $\boldsymbol{R}_{\varepsilon}$ is the covariance matrix $\varepsilon$ and $\boldsymbol{R}$, that of $\boldsymbol{e}$, whose elements are given by variances and covariances of/between the range and bearing estimates, as was shown in Eqs.(7a) through (7f).

From Gaussianity, the probability $P$ that the position estimate in the Cartesian co-ordinates is distributed within a concentration ellipsoid defined by Eq.(10a) around the true source position, is given by Eq.(10b), for any positive $Q$ [6],

$$
\varepsilon^{\tau} R_{\varepsilon}^{-1} \varepsilon \leqq Q, \quad\left(Q \equiv \gamma^{2}, \gamma>0\right) \quad(10 \mathrm{a}) \quad P=2 \int_{0}^{\gamma}(2 \pi)^{-1 / 2} \exp \left(-t^{2} / 2\right) \mathrm{d} t-2 \gamma(2 \pi)^{-1 / 2} \exp \left(-\gamma^{2} / 2\right) .
$$

Therefore, the concentration ellipsoid for a given $Q$, say 4 , provides the expected 3D-distribution area of the proposed source position estimate with probability $P=0.74$, resulting in $3 D$-biaural localization of the source to this prescribed tolerance, so far as the proposed estimate obeys to a 3D-unbiased Gaussian distribution.

By the way, the directions of principal axes of the ellipsoid agree with those of eigen vectors $u k(k=1,2,3)$ of the covariance matrix $R_{\varepsilon}$ and their magnitudes are given by squared root of products of the corresponding eigen values $\lambda_{k s}$ and $Q$. Thus, acoording to the $S V D$ (singular value decomposition) of $\mathbb{R} \varepsilon$, we can straightforwardly evaluate the expected 3D-distribution area of the proposed estimate. Especially, one useful bound for the area may be accessed simply by length of the longest axis of concentration ellipsoids, and it may become a simple practical measure for evaluation of the proposed biaural localization.

\section{RESULTS OF NUMERICAL ANALYSIS}

All the sampling characteristics of the range and bearing estimates in Eqs.(7a) through (7f) include terms that diverge to infinity with the source range ro, since the order of ro in the numerators is greater than in the denominator. This reflects the fact that the information about amplitude attenuation of propagated waves is gradually lost as increase of $r$, since the assumed spherical wave propagation tends to the planer one in the limit, i.e., in the far-field.

Details of the near-field characteristics, however, can not be fully understood from these theoretical considerations, thus necessitating a detailed evaluation, based on numerical analyses, for practical situations. To make clear the fundamentals, some numerical analyses were carried out under the following main conditions:

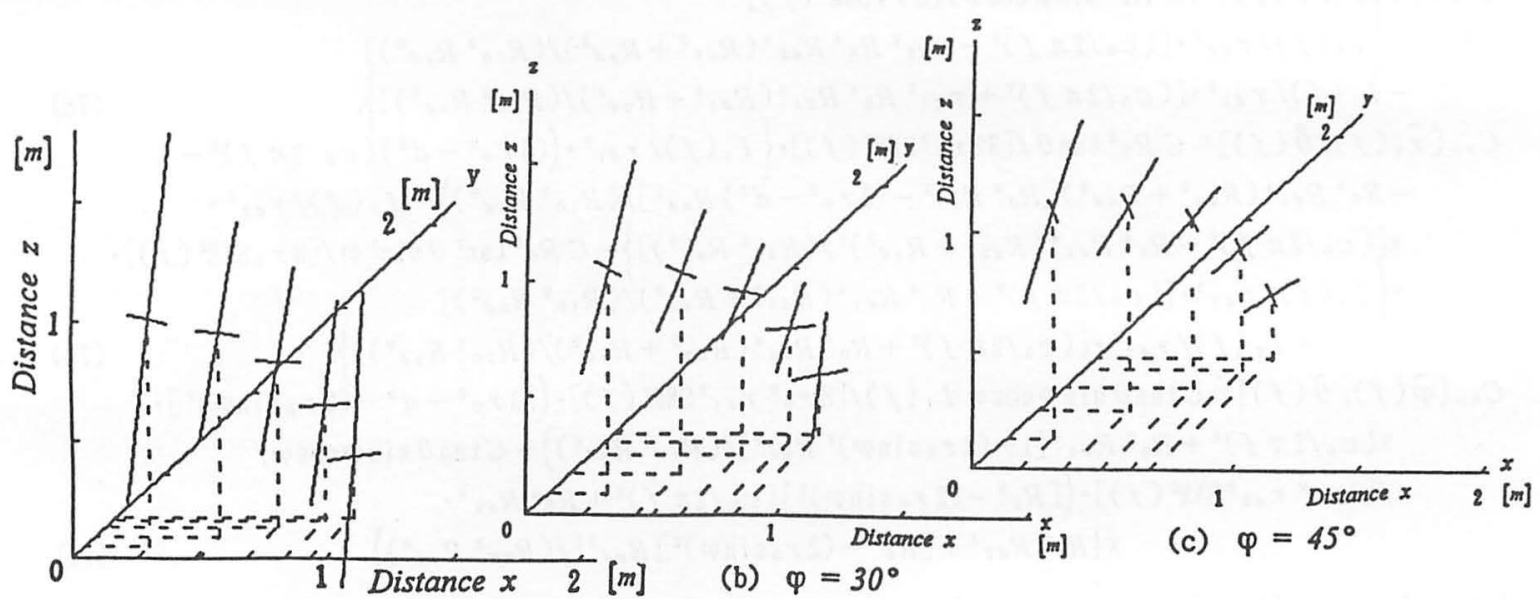

(a) $\varphi=15^{\circ}$

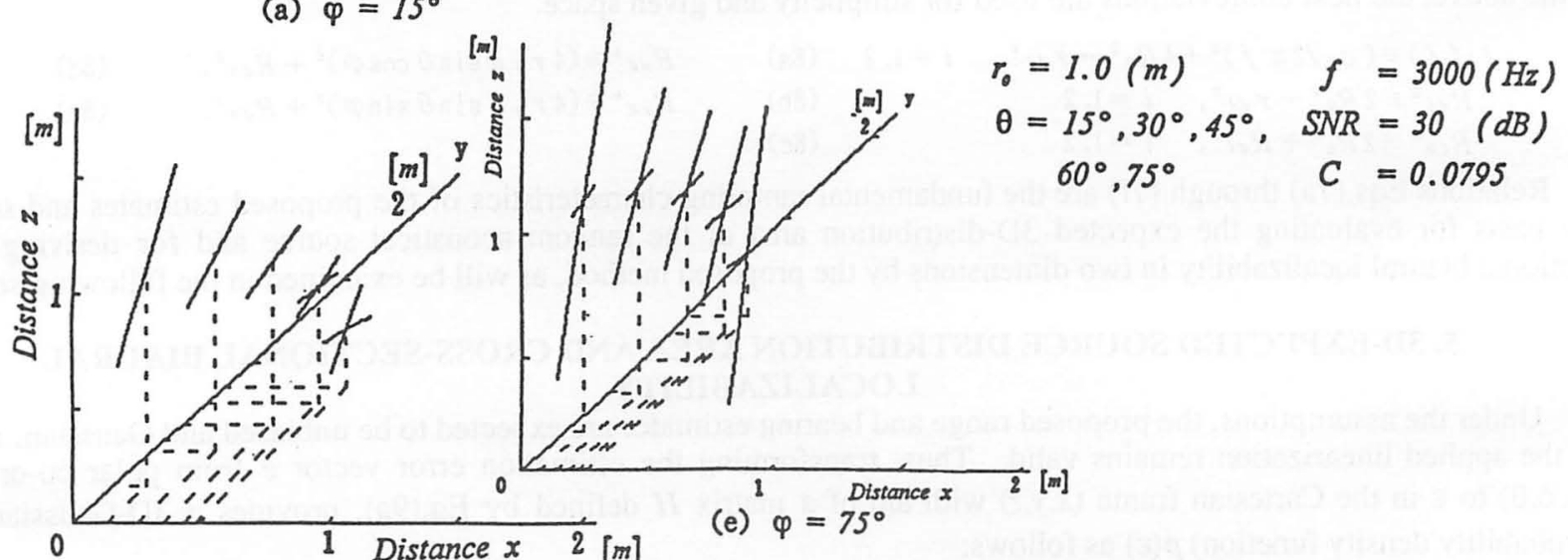

(d) $\varphi=60^{\circ}$

Fig.2 Principal axis representation of evaluated concentration ellipsoids. 
(1) Microphones' separation: $2 d=0.2 \mathrm{~m}$, (2) Source Position: Range; ro $=0.5 \sim 5.0 \mathrm{~m}$, Bearings; $\phi, \theta=5 \sim 85 \mathrm{deg}$.,

(3) Frequency: $f=0.3 \sim 10.0 \mathrm{kHz}$, (4) Signal-to-noise ratio: $S N R=20,30,40 \mathrm{~dB}$, (5) Used windows' parameter:

Bohman's; $C=0.0263$, Hamming's; $C=0.0795$, (6) Concentration ellipsoid parameter: $Q=4$

Fig. 2 through 4 show some typical examples of evaluated concentration ellipsoid for the proposed estimates in the $(x, y, z)$ space. In these figures, three principal axes, perpendicular one another, are shown with line segments. In Fig. 2, $\theta$ and $\phi$ are parametrically changed with $2 d=0.2 \mathrm{~m}, r_{o}=1 \mathrm{~m}, c_{0}=340 \mathrm{~m} / \mathrm{s}, S N R=30 \mathrm{~dB}, f=3 \mathrm{kHz}, Q=4$ and $C=0.795$ being fixed. These results reveal that (1) length lmax of the longest principal axis becomes short as $\phi$ approaches to 45 deg. and (2) concentration ellipsoid exhibits symmetric pattern around $\phi=45$ deg., and (3) within the small region of $\theta$, lmax decreases with increase of $\theta$. The 1 st two features well match with choice of the detector rotation angle being $\alpha=\pi / 2$, since in this case the most effective information detection can be expected at $\phi=45 \mathrm{deg}$., due to the uniform information extraction on differences of propagation distances and amplitude attenuation of propagated waves before and after the rotation. The last feature can be explained as the result of degradation in the precision of range estimate at low bearing $\theta$, as was made clear in the previous paper [1].

By considering the above features, $\theta$ and $S N R$ are parametrically changed, with the other parameters being fixed in Fig.3, while $r_{o}$ is changed with fixed $S N R=30 \mathrm{~dB}$ and $\theta=45 \mathrm{deg}$.. These results clearly show that (4) the precision of the proposed position estimate rapidly decreases with the source range ro, as was considered previously, and (5) its sensitivity to SNR is very high, showing the necessity of high SNR detection.

From the results in Figs. 2 through 4, it is clear that the principal axis of maximum length is extraordinarily dominant, in comparison to the other two. This special feature reveals the important nature that the expected 3D-

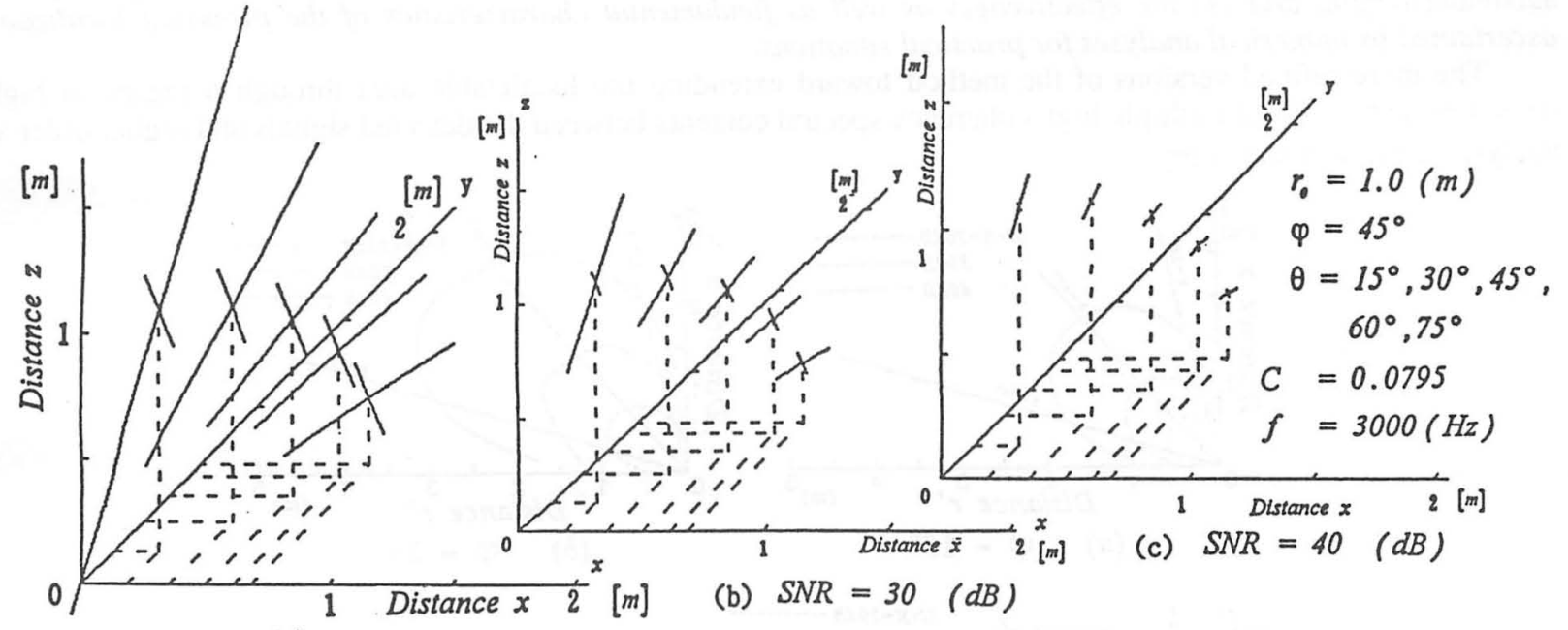

(a) $S N R=20 \quad(d B)$

Fig. 3 Principal axis representation of evaluated concentration ellipsoids.

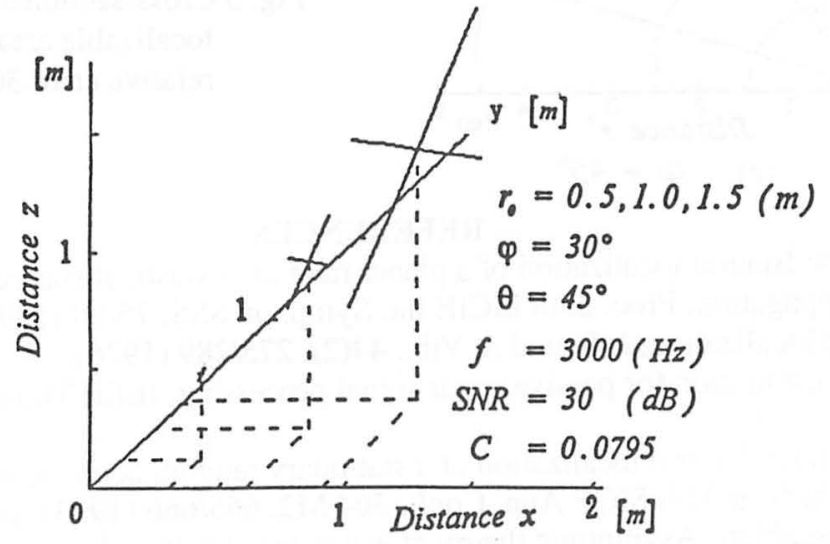

Fig. 4 Principal axis representation of evaluated concentration ellipsoids. 
distribution area of the proposed estimates can be evaluated, simply by a scalar variable, that is, a STDV of the normalized position estimate, with a scale relative to the source range, along the axis, as was theoretically discussed previously.

Three examples of cross-sectional versions of thus derived 3D-localizable area are shown in Fig. 5, where the 3Dareas are examined in the plane normal to the $(x, y)$ one with the source bearing $\phi$ being constant under the assumed condition of using Hamming window and setting the relative error at $30 \%$. As either SNR increases from $20 \mathrm{~dB}$ to $40 \mathrm{~dB}$ or the source bearing $\phi$ approaches to $45 \mathrm{deg}$., the localizable area rapidly extends, for example, from an area within about $\left(z=0.6 \mathrm{~m}, r^{\prime}=0.6 \mathrm{~m}\right)$ in case of $S N R=20 \mathrm{~dB}$ and $\phi=15 \mathrm{deg}$. to $\left(z=3 \mathrm{~m}, r^{\prime}=4.5 \mathrm{~m}\right)$ in case of $S N R=40 \mathrm{~dB}$ and $\phi=45 \mathrm{deg}$.. It should be noted that these areas are derived by supposing the worst case, so that the actual area may be more extended than those shown in the figure, but the degree of extension is different from the source position to position.

\section{CONCLUSIONS}

With aims of exploring humankind's biaural localization ability as well as developing refined devices for environmental recognition in robotics, 3D-biaural localization of a stationary random acoustical source in nesr-fild was studdied and the following results were obtained:

(1) A practical method for simultaneous range and bearing estimation of the random near-field source in the $3 D$ space is proposed by newly introducing a rotation of detectors, in addition to the previously proposed method for planer localization, using the amplitude attenuation of wave propagation and the conventional relative time delay between detected signals. (2) After making clear the principle and the required conditions of the proposed method, (3) 3Dsource distribution or biaural localibable area with prescribed relative error is derived, by way of SV analysis of the theoretically evaluated covariance matrix, as a STDV of the normalized position estimate along the principal axis of maximum length, and (4) the effectiveness as well as fundamental characteristics of the proposed localization are ascertained by numerical analyses for practical situations.

The more refined versions of the method toward extending the localizable area through a means of high SNR detection such as use of multiple high coherency spectral contents between the detected signals and higher order spectral analyses are now under study.

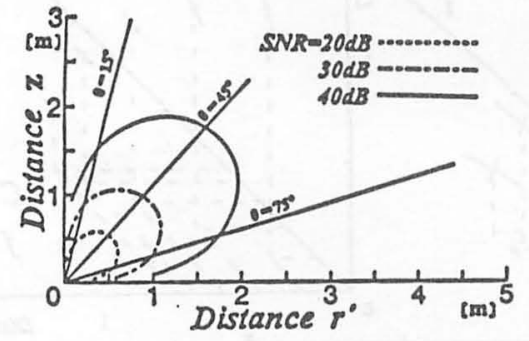

(a) $\varphi=15^{\circ}$

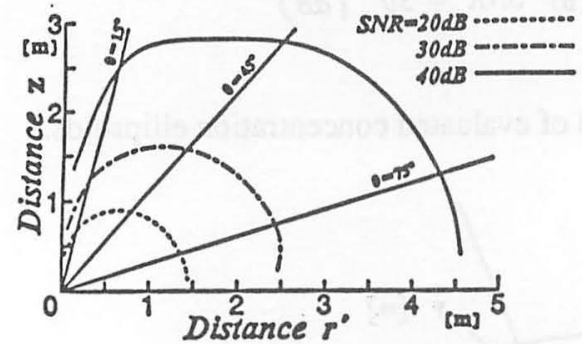

(c) $\varphi=45^{\circ}$

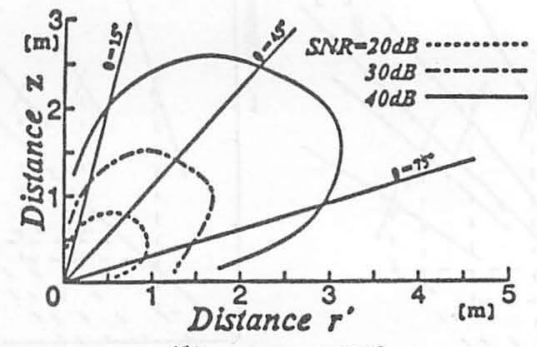

(b)

$$
\begin{aligned}
& f=3000 \mathrm{~Hz} \\
& C=0.0795
\end{aligned}
$$

Fig. 5 Cross-sectional biaural localizable area with relative error $30 \%$

\section{REFERENCES}

[1] K. Sasaki \& K. Hirasawa: Biaural localization of a planer random acoustical source in near field using amplitude attenuation of wave propagation, Proc. 25th ISCIE Int. Symp. on SSS, 75/80 (1994).

[2] R. Kinns: Biaural source localization, J. Sound \& Vib., 44(2), 275/289 (1976).

[3] G.C. Carter: Time delay estimation for passive sonar signal processing, IEEE Trans. ASSP, ASSP-29(3), $463 / 469$ (1981).

[4] K. Sasaki: Three dimensional biaural localization of a stationary random acoustic source in near field with additional rotation of detectors, Preprint 32th SICE Ann. Conf., 304 M2, $665 / 666$ (1993) (in Japanese).

[5] D.R. Brillinger \& M. Rosenblatt: Asymptotic theory of estimates of k-th order spectra, in Time Series Analysis, B. Harris, Ed., John Wiley, New York (1967).

[6] H.L. Van Trees: Detection, Estimation and Modulation Theory Vol. I, John Wiley, New York (1967). 